\title{
Propriedades relevantes para a otimização da secagem de concretos refratários
}

\section{(Key properties for the optimization of drying refractory castables)}

\author{
M. M. Akiyoshi, M. D. M. Innocentini, F. A. Cardoso, V. C. Pandolfelli \\ Universidade Federal de $S$. Carlos \\ Rod. Washington Luiz, km 235, C.P. 676, S. Carlos, SP, 13565-905 \\ pmmak@iris.ufscar.br,vicpando@power.ufscar.br
}

\begin{abstract}
Resumo
A necessidade de redução do consumo energético tem impulsionado a busca por concretos refratários que possibilitem a diminuição do seu tempo de secagem. Todavia, a secagem de concretos refratários é uma das etapas mais críticas de seu processamento. Condições não otimizadas de aquecimento podem gerar riscos consideráveis de explosão. Dentre os fatores que podem afetar esses riscos, a cura em baixas temperaturas tem sido descrita como um fator de grande influência. No entanto, em trabalhos anteriores, os aspectos de permeabilidade, ensaios de explosão ou mecânicos têm sido avaliados isoladamente. Este estudo avalia e discute, de forma sistêmica, o efeito da permeabilidade, da cinética de saída de água, da resistência mecânica e dos perfis de temperatura desenvolvidos na superfície dos corpos-de-prova curados em temperaturas distintas $\left(8{ }^{\circ} \mathrm{C}\right.$ e $\left.50{ }^{\circ} \mathrm{C}\right)$.

Palavras-chave: concreto refratário, secagem, permeabilidade, perfis de temperatura.
\end{abstract}

Abstract

The increasing demand for better and cheaper refractory products has motivated the search for safer and shorter drying schedules in the refractory industry. If the drying process is not carefully controlled, the risk of explosive spalling increases considerably. The curing at low temperatures has been pointed as one of the most important factors. Nevertheless, so far, permeability, mechanical resistance and explosion tests has been evaluated separately. This work aims to correlate the former mentioned properties with the dewatering and temperature profile of castables cured at $8{ }^{\circ} \mathrm{C}$ and $50^{\circ} \mathrm{C}$.

Keywords: refractory castable, drying, permeability

\section{INTRODUÇÃO}

A crescente demanda por concretos refratários melhores e mais baratos tem motivado a busca por programas de secagem mais seguros e otimizados. Todavia, a secagem é uma das etapas mais complexas do processamento destes materiais, uma vez que envolve gastos com energia, com o tempo de parada de equipamentos e, quando não realizada de forma cuidadosa, pode acarretar graves acidentes ou até mesmo explosões.

Baixas temperaturas de cura (menores que $20^{\circ} \mathrm{C}$ ) [1] têm sido descritas como um dos fatores de grande influência na ocorrência de explosões. Uma análise mais detalhada, entretanto, revela que a temperatura de cura afeta variáveis [2], tais como a resistência mecânica, a permeabilidade e a condutividade térmica, propriedades estas, até o momento, avaliadas de forma isolada.

Outros trabalhos $[3,4]$ estudaram a saída de água livre e os perfis de temperatura interna e perda de massa durante o processo de secagem de concretos pré-queimados utilizando diversas taxas de aquecimento.

Neste contexto, este trabalho avalia de forma sistêmica a resistência mecânica e a permeabilidade de concretos a verde buscando correlacioná-las com os perfis de perda de massa e temperatura de concretos curados a $8{ }^{\circ} \mathrm{C}$ e $50{ }^{\circ} \mathrm{C}$.

\section{PROCEDIMENTO EXPERIMENTAL}

Materiais utilizados: empregaram-se concretos refratários auto-escoantes, de alta alumina, com ultra-baixo teor de cimento (2\%-p) preparados seguindo-se o modelo de distribuição de tamanho de partículas de Andreasen ( $\mathrm{q}=0,21)$. Os concretos utilizados possuíam uma matriz fina composta por aluminas calcinadas (A1000 SG e A3000 FL) e pelo cimento de aluminato de cálcio (CA14). A fração grossa era composta por agregados de alumina eletrofundida branca, na faixa de $100 \mu \mathrm{m}-5600 \mu \mathrm{m}$ totalizando $99 \%$-p de $\mathrm{Al}_{2} \mathrm{O}_{3}$ e $0,6 \%$-p de $\mathrm{CaO}$. Todas as matérias-primas foram fornecidas pela Alcoa Alumínio.

Temperatura e tempo de cura foram alterados para produzir modificações na permeabilidade e na resistência mecânica dos concretos sem alterar significativamente a condutividade térmica. Os dois tempos de cura utilizados foram 2 dias (comumente empregado industrialmente) e 16 dias para permitir uma maior hidratação do concreto curado a $8{ }^{\circ} \mathrm{C}[6,7]$. Considerando-se que todos os concretos neste trabalho foram produzidos a partir das mesmas matérias-primas e que a ação da temperatura de cura atua apenas sobre o cimento (que é o componente minoritário, apenas $2 \% \mathrm{p}$ ), assumiu-se que a condutividade térmica dos concretos curados nas duas temperaturas seja 
constante. Resultados anteriores e internos do grupo dos autores mostraram pouca diferença entre as porosidades aparente e total para os concretos curados a $8{ }^{\circ} \mathrm{C}$ e $50{ }^{\circ} \mathrm{C}$.

Os concretos foram moldados na forma de cilindros com $7,5 \mathrm{~cm}$ de diâmetro e $2,5 \mathrm{~cm}$ de espessura para os ensaios de permeabilidade e cilindros de $4,0 \mathrm{~cm}$ de diâmetro e $4,0 \mathrm{~cm}$ de altura para os ensaios de análise termogravimétrica (ATG), determinação do perfil de temperatura interna e avaliação da resistência mecânica à tração por compressão diametral. A secagem das amostras para avaliação da permeabilidade e da resistência mecânica foi realizada na temperatura de cura utilizando-se sílica gel.

Resistência mecânica: a resistência mecânica à tração dos concretos foi determinada através de um ensaio de compressão diametral utilizando-se uma taxa de carregamento de $42 \mathrm{~N} / \mathrm{s}$ de forma a garantir que a taxa de aumento de tensão se mantivesse entre $690 \mathrm{kPa} / \mathrm{min}$ e $1380 \mathrm{kPa} / \mathrm{min}$ [7]. Para cada um dos concretos foram avaliados 5 corpos-de-prova. A tensão de ruptura por compressão diametral é dada por:

$$
\sigma=\frac{2 \cdot P}{\pi \cdot h \cdot d}(\mathrm{MPa}),
$$

na qual, $\mathrm{P}(\mathrm{N})$ é a carga de ruptura, d (mm) e h (mm) são, respectivamente, o diâmetro e a altura do corpo-de-prova.

Medida de permeabilidade em temperatura ambiente: a avaliação da permeabilidade foi realizada em amostras secas empregando-se ar como fluido permeante. $\mathrm{O}$ ajuste das constantes Darciana (k1) e não-Darciana (k2) foi efetuado através da equação de Forchheimer [8] para fluidos compressíveis expressa por:

$$
\frac{P_{\mathrm{e}}^{2}-P_{s}^{2}}{2 \cdot P_{s} L}=\frac{\mu}{k_{1}} \cdot v_{s}+\frac{\rho}{k_{2}} \cdot v_{s}^{2}
$$

na qual, $\mathrm{P}_{\mathrm{e}}$ e $\mathrm{P}_{\mathrm{s}}$ são as pressões absolutas na entrada e na saída da amostra, respectivamente; $\mu$ é a viscosidade; $\rho$ é a densidade do fluido; L é a espessura da amostra; e $\mathrm{v}_{\mathrm{s}}$ é a velocidade do fluido (dada pela razão entre a vazão volumétrica e a área da seção transversal perpendicular ao escoamento do fluido). Os ensaios foram realizados em réplica.

Análise termogravimétrica (ATG): o equipamento de análise termogravimétrica [4] consiste em uma balança eletrônica $(400 \pm 0,001 \mathrm{~g})$ acoplada a um forno tipo mufla (temperatura máxima de trabalho de $1100{ }^{\circ} \mathrm{C}$ ). $\mathrm{O}$ forno possui um controle de temperatura que permite a aplicação de taxas de aquecimento de $0,1{ }^{\circ} \mathrm{C} / \mathrm{min}$ até $20{ }^{\circ} \mathrm{C} / \mathrm{min}$. Um programa de computador dedicado possibilita o monitoramento simultâneo da perda de massa, da temperatura no forno e da temperatura na superfície do corpo-de-prova.

A perda de massa foi acompanhada através do parâmetro adimensional $\mathrm{W}$, que expressa a razão entre a massa de água perdida no instante i e a massa total de água existente no corpode-prova, sendo expresso por:
Perda de massa:

$$
\mathrm{W}=100 \cdot \frac{\mathrm{m}_{0}-\mathrm{m}_{\mathrm{i}}}{\mathrm{m}_{0}-\mathrm{m}_{\mathrm{f}}}(\%,
$$

A taxa de perda de massa (dW/dt) foi calculada derivandose $\mathrm{W}$ em relação ao tempo:

Taxa de perda de massa:

$$
\left(\frac{d W}{d t}\right)=\frac{d}{d t}\left(\frac{m_{0}-m_{i}}{m_{0}-m_{f}}\right)(\% \min ),
$$

A quantidade total de água determinada experimentalmente (em base seca) $\mathrm{W}_{\mathrm{D}}$ deve ser aproximadamente igual à quantidade adicionada durante a mistura $\left(\mathrm{W}_{\text {mistura }}=4,50 \%-\mathrm{p}\right)$ do concreto, podendo ser expressa por:

Quantidade total de água (base seca):

$$
W_{D}=100 \cdot \frac{m_{0}-m_{f}}{m_{f}}(\%-p)
$$

nas quais: $\mathrm{m}_{0}$ é a massa inicial, $\mathrm{m}_{\mathrm{f}}$ é a massa final e $\mathrm{m}_{\mathrm{i}}$ é a massa no instante $i$

Determinação do perfil de temperatura interna. A determinação da temperatura interna na superfície dos refratários foi realizada através de um termopar, posicionado sobre a linha geratriz do corpode-prova, próximo à superfície (cerca de $1 \mathrm{~mm}$ abaixo). A taxa de aquecimento foi obtida a partir da derivada da temperatura na superfície em relação ao tempo como apresentado pela equação $\mathrm{F}$.

Taxa de perda de massa:

$$
\left(\frac{d T_{\text {sup }}}{d t}\right)_{i}=\left(\frac{T_{\text {sup }_{i+1}}-T_{\text {sup }_{i-1}}}{t_{i+1}-t_{i-1}}\right)\left({ }^{0} \mathrm{C} / \text { min }\right)
$$

na qual: $\mathrm{T}_{\text {sup }}$ é a temperatura na superfície e t é o tempo.

Para a avaliação da tendência à explosão foi empregada uma taxa de aquecimento no forno de $20{ }^{\circ} \mathrm{C} / \mathrm{min}$ enquanto que os perfis de perda de massa e de temperatura interna foram obtidos com uma taxa de $10{ }^{\circ} \mathrm{C} / \mathrm{min}$.

\section{RESULTADOS E DISCUSSÃO}

Os concretos curados a $8{ }^{\circ} \mathrm{C}$ e $50{ }^{\circ} \mathrm{C}$ serão denominados concreto-8 e concreto-50, respectivamente. Os ensaios de explosão realizados com uma taxa de $20{ }^{\circ} \mathrm{C} / \mathrm{min}$ mostraram que apenas os concretos-8 (independentemente do tempo de cura) explodiram, confirmando a maior tendência à explosão de concretos curados em baixas temperaturas. A temperatura de explosão, a resistência mecânica e as constantes de permeabilidade são apresentadas na Tabela I. Nota-se que a temperatura e o tempo de cura influenciam tanto a permeabilidade 
Tabela I - Tempo e temperatura de cura, temperatura de explosão (taxa de aquecimento de $20^{\circ} \mathrm{C} / \mathrm{min}$ ), resistência mecânica a seco $(\sigma)$ e constantes de permeabilidade $\left(\mathrm{k}_{1}\right.$ e $\left.\mathrm{k}_{2}\right)$ dos concretos avaliados.

[Table I-Curing conditions, explosion temperature (heating rate of $20^{\circ} \mathrm{C} / \mathrm{min}$ ), tensile mechanical strength ( $\left.\sigma\right)$, and permeability constants $\left(k_{1}\right.$ and $k_{2}$ ) for the castables evaluated.]

\begin{tabular}{cccccc}
\hline \multirow{2}{*}{ cura (dias) } & $\begin{array}{c}\text { Temperatura } \\
\text { de cura }\left({ }^{\circ} \mathrm{C}\right)\end{array}$ & $\begin{array}{c}\text { Temperatura de } \\
\text { explosão }\left({ }^{\circ} \mathrm{C}\right)\end{array}$ & $\begin{array}{c}\sigma \\
(\mathrm{MPa})\end{array}$ & $\begin{array}{c}\mathrm{K}_{1} \\
\left(10^{-16} \mathrm{~m}^{2}\right)\end{array}$ & $\begin{array}{c}\mathrm{K}_{2} \\
\left(10^{-16} \mathrm{~m}^{2}\right)\end{array}$ \\
\hline 2 & 8 & $426 \pm 3$ & $1,3 \pm 0,1$ & $2,4 \pm 0,1$ & $176 \pm 17$ \\
\cline { 2 - 6 } & 50 & sem explosão & $2,0 \pm 0,3$ & $3 \pm 1$ & $311 \pm 23$ \\
\hline \multirow{2}{*}{16} & 8 & $440 \pm 7$ & $2,9 \pm 0,4$ & $1,0 \pm 0,1$ & $19 \pm 1$ \\
\cline { 2 - 6 } & 50 & sem explosão & $2,0 \pm 0,2$ & $2,2 \pm 0,1$ & $189 \pm 23$ \\
\hline
\end{tabular}

quanto a resistência mecânica dos concretos [5, 6].

Para 2 dias de cura, nota-se que o concreto 8 apresentou resistência mecânica inferior ao concreto 50 . Segundo a literatura $[9,11]$, a cura em temperaturas menores que $20{ }^{\circ} \mathrm{C}$ favorece a formação de hidratos de baixa densidade, principalmente o $\mathrm{CaO} \cdot \mathrm{Al}_{2} \mathrm{O}_{3} \cdot 10 \cdot \mathrm{H}_{2} \mathrm{O}\left(\mathrm{CAH}_{10}, \rho=1,72 \mathrm{~g} / \mathrm{cm}^{3}\right)$ e alumina gel que podem gerar estruturas resistentes, mas pouco permeáveis. Temperaturas superiores favorecem a formação de hidratos mais densos, como $2 \mathrm{CaO} \cdot \mathrm{Al}_{2} \mathrm{O}_{3} \cdot 8 \mathrm{H}_{2} \mathrm{O}$ $\left(\mathrm{C}_{2} \mathrm{AH}_{8}, \rho=1,95 \mathrm{~g} / \mathrm{cm}^{3}\right), 3 \mathrm{CaO} \cdot \mathrm{Al}_{2} \mathrm{O}_{3} \cdot 6 \mathrm{H}_{2} \mathrm{O}\left(\mathrm{C}_{3} \mathrm{AH}_{6}, \rho=2,52 \mathrm{~g} / \mathrm{cm}^{3}\right)$ e $\mathrm{Al}(\mathrm{OH})_{3}\left(\mathrm{AH}_{3}, \rho=2,42 \mathrm{~g} / \mathrm{cm}^{3}\right)$ que tendem originar estruturas mais permeáveis, todavia, menos resistentes.

A temperatura de cura afeta a cinética de hidratação do cimento [5,6], que é o principal agente responsável pela resistência mecânica do concreto a verde. Embora a cura em baixas temperaturas favoreça a formação de estruturas mais resistentes, $\mathrm{o}$ menor valor de resistência mecânica para a temperatura de $8{ }^{\circ} \mathrm{C}$ após 2 dias de cura pode ser atribuído à menor velocidade de hidratação do cimento em baixas temperaturas. Essa conclusão é reforçada quando se observa que após 16 dias, o concreto 8 se torna mais resistente que o concreto curado em temperatura superior. Devido a sua maior velocidade de hidratação, o concreto 50 praticamente atinge o máximo de sua resistência mecânica após 2 dias, enquanto o concreto 8 continua a aumentar sua resistência à medida que a hidratação prossegue.

Nota-se que as constantes de permeabilidade $\mathrm{k}_{1}$ e $\mathrm{k}_{2}$ são menores para os concretos curados a $8{ }^{\circ} \mathrm{C}$. Apesar do concreto 8 apresentar menor grau de hidratação que o concreto 50 , as fases de baixa densidade acarretam uma estrutura menos permeável e mais resistente. Essa redução na permeabilidade e aumento na resistência mecânica são acentuados à medida que o tempo de cura aumenta para 16 dias, uma vez que a quantidade destas fases cresce com o tempo de cura.

Comparando-se os concretos curados a $8{ }^{\circ} \mathrm{C}$ por 2 e 16 dias, nota-se que embora o concreto curado por 16 dias apresente permeabilidade menor, sua maior resistência mecânica causou um aumento na temperatura de explosão de $426{ }^{\circ} \mathrm{C}$ para $440{ }^{\circ} \mathrm{C}$. Contudo, ao se analisar os concretos curados a $8{ }^{\circ} \mathrm{C}$ e $50{ }^{\circ} \mathrm{C}$ por 16 dias nota-se que embora o concreto curado 8 seja mais resistente, sua falha ao ser submetido a uma taxa de aquecimento de $20{ }^{\circ} \mathrm{C} /$ min pode ser atribuída a sua baixa permeabilidade $\left(\mathrm{k}_{2}\right.$ é uma ordem de grandeza maior para o concreto 50).

Uma correlação entre a resistência mecânica, a permeabilidade e a tendência à explosão para as temperaturas de cura de $8{ }^{\circ} \mathrm{C} e$ $50^{\circ} \mathrm{C}$ pode ser melhor visualizada quando são analisados os perfis de perda de massa e de temperatura na superfície.

A secagem de concretos refratários pré-queimados, saturados e submetidos a uma taxa de aquecimento constante pode ser dividida em três estágios [5]. O primeiro e segundo estágios estão relacionados, respectivamente, à evaporação e à ebulição da água livre, enquanto o terceiro estágio se relaciona a processos de desidratação da água combinada proveniente dos hidratos formados pelo cimento e aluminas reativas.

A mesma associação foi utilizada para a análise dos perfis de taxa de perda de massa e temperatura na superfície dos concretos curados a $8^{\circ} \mathrm{C} \mathrm{e} 50^{\circ} \mathrm{C}$ a verde. Todavia, em se tratando de concretos a verde, os perfis de evaporação e ebulição podem contar com a contribuição da saída do vapor proveniente da decomposição dos hidratos, não sendo possível associar as perdas de massa com as quantidades de água liberada na evaporação, ebulição ou desidratação, separadamente.

A Fig. 1a apresenta as curvas da perda de massa (W) e sua derivada $(\mathrm{dW} / \mathrm{dt})$ enquanto na Fig. $1 \mathrm{~b}$ tem-se a taxa de aquecimento na superfície $\left(\mathrm{dT}_{\text {sup }} / \mathrm{dt}\right)$ para os concretos curados a $8^{\circ} \mathrm{C}$ e $50^{\circ} \mathrm{C}$ por 2 dias ensaiados com uma taxa de aquecimento média de $10^{\circ} \mathrm{C} / \mathrm{min}$ (indicada por uma linha tracejada).

A taxa de perda de massa é um balanço entre fatores que favorecem a saída de água (tal como a evaporação, a ebulição e a saída de hidratos) e a retração da frente de secagem para o interior do refratário que tende a dificultar a saída de água [4]. A quantidade de água perdida em cada etapa pode ser avaliada experimentalmente através da diferença dos valores de $\mathrm{W}$ da região que compreende dois vales consecutivos na curva dW/dt. Esses são apresentados na Tabela II.

Comparado com o concreto 8 que possuía 4,49\% p de água, o concreto 50 apresenta uma quantidade total de água $(4,36 \% \mathrm{p})$ inferior a adicionada durante a mistura $(4,50 \% \mathrm{p})$, pois mesmo realizando-se a cura em ambiente saturado, observa-se que há perda de água para o meio em temperaturas de cura mais elevadas, possivelmente devido à diminuição da viscosidade da água e ao aumento da energia cinética de suas moléculas.

No estágio I (Fig. 1a) predomina a evaporação, uma vez que se têm temperaturas inferiores a $100^{\circ} \mathrm{C}$. Como a secagem se realiza do 
Tabela II - Quantidade total de água (base seca), temperatura de pico $\left(\mathrm{T}_{\mathrm{i}}\right)$ e quantidade de água perdida $\left(\mathrm{W}_{\mathrm{i}}\right)$ em cada estágio ( $\mathrm{i}=1,2$ ou 3 , para os estágios I, II ou III, respectivamente) para os concretos curados a $8{ }^{\circ} \mathrm{C}$ e $50^{\circ} \mathrm{C}$ por 2 dias ensaiados com uma taxa de $10{ }^{\circ} \mathrm{C} / \mathrm{min}$ (resultados obtidos na Fig. 1a).

[Table II - Total amount of water (dry-basis), peak temperature (T), and mass loss (W) for each stage ( $i=1,2$ or 3 for stages I, II or III, respectively) for castables cured at $8{ }^{\circ} \mathrm{C}$ or $50{ }^{\circ} \mathrm{C}$ for 2 days (results based on Fig. la).]

\begin{tabular}{lccccccc}
\hline $\begin{array}{l}\text { Temperatura } \\
\text { de cura }\left({ }^{(} \mathrm{C}\right)\end{array}$ & $\mathrm{W}_{\mathrm{d}}$ & $\mathrm{T}_{1}$ & $\mathrm{~W}_{1}$ & $\mathrm{~T}_{2}$ & $\mathrm{~W}_{2}$ & $\mathrm{~T}_{3}$ & $\mathrm{~W}_{3}$ \\
\hline 8 & $(\%)$ & $\left({ }^{\circ} \mathrm{C}\right)$ & $(\%)$ & $\left({ }^{\circ} \mathrm{C}\right)$ & $(\%)$ & $\left({ }^{\circ} \mathrm{C}\right)$ & $(\%)$ \\
\hline 50 & 4,49 & 75 & 38 & 189 & 55 & 363 & 7 \\
\hline
\end{tabular}

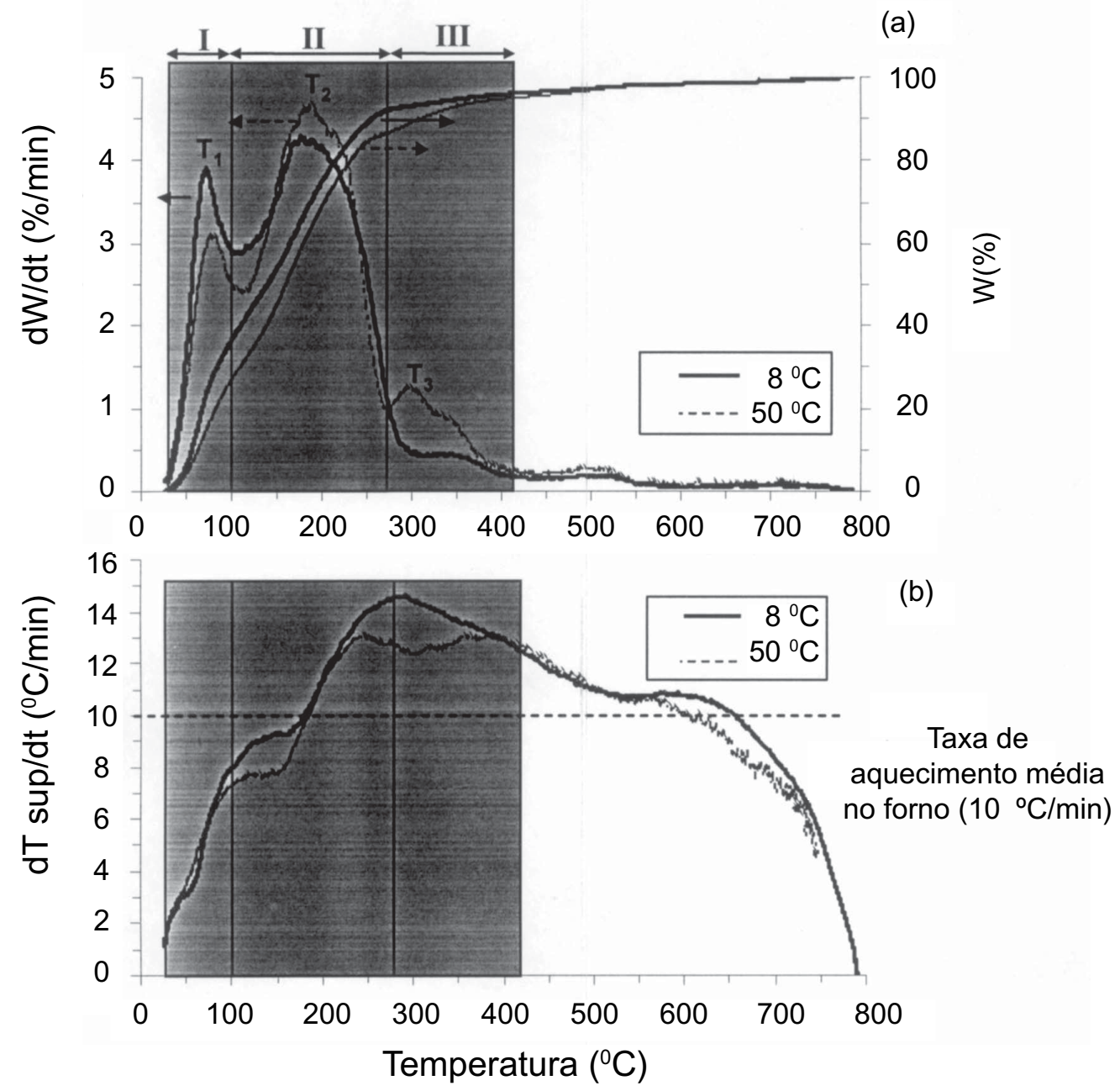

Figura 1: (a) perda de massa (W) e sua derivada (dW/dt) e (b) taxa de aquecimento na superfície $\left(\mathrm{dT}_{\text {sup }} / \mathrm{dt}^{\mathrm{d}}\right)$ para concretos curados a $8{ }^{\circ} \mathrm{C}$ e $50{ }^{\circ} \mathrm{C}$ por 2 dias ensaiado a uma taxa de $10{ }^{\circ} \mathrm{C} / \mathrm{min}$.

[Figure 1: (a) mass loss $(W)$ and mass loss rate (dW/dt), (b) surface heating rate $\left(d T_{\text {sup }} / d t\right)$ for castables cured at $8{ }^{\circ} \mathrm{C}$ and $50{ }^{\circ} \mathrm{C}$ for 2 days and evaluated at a heating rate of $10^{\circ} \mathrm{C} / \mathrm{min}$.]

exterior para o interior do refratário, os concretos curados em temperaturas menores tendem perder menos água durante a etapa de cura. Além disso, a menor cinética de hidratação em baixas temperaturas, faz com que uma menor quantidade da água adicionada durante a mistura se encontre na forma de água combinada (que só será liberada em temperaturas superiores a $100{ }^{\circ} \mathrm{C}$ ).

A menor perda de água sofrida durante a cura e a menor cinética de hidratação contribuíram para que o concreto 8 apresentasse maior quantidade de água livre que o concreto 50 . Deste modo, apesar do concreto 8 ser menos permeável, este apresentou taxa de perda de massa e quantidade de água perdida no estágio I $\left(\mathrm{w}_{1}=38 \%\right)$ superiores ao concreto $50\left(\mathrm{w}_{1}=32 \%\right)$.

Nota-se na Fig. $1 \mathrm{~b}$ que durante o estágio $\mathrm{I}$, a taxa de aquecimento na superfície para os concretos curados a $8{ }^{\circ} \mathrm{C}$ e $50^{\circ} \mathrm{C}$ são similares, 
uma vez que durante a evaporação a saída de água é comandada majoritariamente por variáveis externas, tais como a taxa de aquecimento e a umidade relativa. No estágio I, parte do calor recebido pelo corpo-de-prova é utilizado na evaporação da água impedindo que a taxa de aquecimento na superfície dos concretos acompanhe a taxa do forno $\left(10^{\circ} \mathrm{C} / \mathrm{min}\right)$. Neste estágio, praticamente não existe pressurização da estrutura, de forma que mesmo o concreto curado a $8^{\circ} \mathrm{C}$ por 2 dias que apresenta a menor resistência mecânica não sofreria dano caso a secagem se efetuasse abaixo de $100{ }^{\circ} \mathrm{C}$.

Contudo, os efeitos da permeabilidade sobre a perda de massa começam a se tornar mais significativos a partir do estágio II, onde se inicia a ebulição da água (em torno de $100{ }^{\circ} \mathrm{C}$ ), uma vez que a saída de água passa a ser controlada pela pressurização do vapor no interior da estrutura, sendo que esta influência aumenta à medida que a frente de secagem avança para o interior do corpo-de-prova.

A diferença de permeabilidade entre os concretos curados a $8{ }^{\circ} \mathrm{C}$ e $50{ }^{\circ} \mathrm{C}$ faz-se notar pela separação de suas taxas de aquecimento na superfície (Fig. 1b). À medida que a ebulição ocorre, o vapor proveniente do interior do corpo-de-prova refrigera a superfície, impedindo-a de acompanhar a taxa de aquecimento do forno $\left(10{ }^{\circ} \mathrm{C} / \mathrm{min}\right)$. Devido a sua maior permeabilidade, o concreto curado a $50{ }^{\circ} \mathrm{C}$ apresenta uma taxa de aquecimento na superfície inferior ao concreto curado a $8{ }^{\circ} \mathrm{C}$.

A partir do início da ebulição pode ocorrer a pressurização do vapor no interior da estrutura, sendo o aumento de pressão tanto maior quanto menos permeável for o concreto, ocorrendo a falha durante a secagem quando a pressão no interior do refratário se igualar à resistência mecânica do material. Deste modo, quanto maior a resistência mecânica, maior a temperatura de explosão.

Outro fator que favorece a menor tendência à explosão do concreto 50 é a formação durante a cura de uma maior quantidade de hidratos que se decompõem em temperaturas superiores a da saída da água livre. A explosão do concreto 8 , quando aquecido a uma taxa de $20^{\circ} \mathrm{C} / \mathrm{min}$, ocorreu quando a superfície do corpo-de-prova estava a aproximadamente $155{ }^{\circ} \mathrm{C}$ enquanto os hidratos do concreto 50 se decompõem, em sua maioria, em temperaturas acima de $200^{\circ} \mathrm{C}$, como mostra a maior intensidade do terceiro pico $\left(\mathrm{T}_{3}\right)$ de saída de água para este concreto.

Comparando-se as quantidades de água perdida durante o terceiro pico, nota-se que o valor de $\mathrm{w}_{3}=14 \%$ para concreto 50 é superior ao valor para o concreto $8\left(\mathrm{w}_{3}=7 \%\right)$. Este valor de $\mathrm{w}_{350^{\circ} \mathrm{C}}-\mathrm{w}_{38^{\circ} \mathrm{C}}=7 \%$, é aproximadamente a diferença da quantidade de água perdida no primeiro pico pelos dois concretos.

Assim, a análise da resistência mecânica e da permeabilidade em conjunto com os perfis de perda de perda de massa e temperatura interna permitem concluir que a baixa permeabilidade é o principal fator responsável pela maior tendência à explosão de concretos curados em baixas temperaturas, uma vez que favorece não só o aprisionamento de mais água no interior da estrutura (contribuindo para o aumento da pressão interna) como também expõe o corpo-de-prova a maiores taxas de aquecimento.

\section{CONCLUSÕES}

A cura a $8{ }^{\circ} \mathrm{C}$ e $50{ }^{\circ} \mathrm{C}$ causa diferenças significativas na resistência mecânica e na permeabilidade que se refletem nos perfis de perda de massa e taxa de aquecimento na superfície dos concretos. A maior tendência à explosão dos concretos curados em baixas temperaturas pode ser atribuída principalmente à sua baixa permeabilidade, uma vez que esta propriedade esta relacionada não apenas a dificuldade de saída do vapor da estrutura do refratário, como também a taxas de aquecimento mais severas na superfície e maior quantidade de hidratos que se decompõem em temperaturas próximas à temperatura de saída da água livre.

\section{AGRADECIMENTOS}

Os autores agradecem à FAPESP, CAPES, Alcoa S. A. e Magnesita S. A.

\section{REFERÊNCIAS}

[1] W. Gitzen, L. D. Hart, "Explosive spalling of refractory castables bonded with calcium aluminate cement", Am. Ceram. Soc. Bull. 40, 8 (1961) 503 507, 510.

[2] R. E. Moore, J. D. Smith, W. L. Headrick Jr., T. P. Sander, "Monolithic dewatering theory testing and practice: new challenges", in $32^{\text {th }}$ Symp. on Refractories, The Am. Ceram. Soc., St. Louis Section (1996) 26p.

[3] M. D. M. Innocentini, C. Ribeiro, L. R. M. Bittencourt, V. C. Pandolfelli, "Fluid dynamics and thermal aspects of the dewatering of high-alumina refractory castables: removal of physically absorbed water", J. Am. Ceram. Soc. 84, 11 (2001) 2696-2698.

[4] M. D. M. Innocentini, F. A. Cardoso, M. M. Akiyoshi, V. C. Pandolfelli, "Drying stages during the heat-up of high alumina, ultra-low cement refractory castables", J. Am. Ceram. Soc. 86, 7 (2003) 1146-1148.

[5] F. A. Cardoso, M. M. Akiyoshi, M. D. M. Innocentini, V. C. Pandolfelli, "Effect of curing time on the properties of CAC bonded refractory castables", J. Eur. Ceram. Soc. 24, 7 20732078 (2004) 2073-2078.

[6] M. R. Nilforoushan, J. H. Sharp, "The Effect of Time on The hydration reactions of a refractory calcium aluminate cement investigated by SEM", in Proc. Unified Int. Technical Conf. on Refractories - UNITECR'2001 ALAFAR, Cancun (2001) pp.1586-1597.

[7] American Society for Standardization. C 496-90. "Standard test method for splitting tensile strength of cylindrical concrete specimens", v. 04.02 .

[8] M. D. M. Innocentini, V. C. Pandolfelli, “Considerações sobre a estimativa da permeabilidade em concretos refratários através das equações de Darcy e de Forchheimer", Cerâmica 45, 292293 (1999) 61-67.

[9] G. MacZura, L. D. Hart, R. O, Heilich, J. E. Kopanda, "Refractory Cement"; Ceramic, in Proc. Am. Ceram. Soc. (1983) 11p.

[10] K. M. Parker, J. H. Sharp, "Refractory Calcium Aluminate Cements", Trans. J. Brit. Ceram. Soc. 81 (1982) 35-42.

[11] A. Nishikawa, Technology of Monolithic Refractories, Plibrico Japan Co., Ltd., Tokyo, Japan (1984) 83-170.

(Rec. 20/06/03, Ac. 03/10/03) 